\title{
Erratum to: A new schedule of fotemustine in temozolomide- pretreated patients with relapsing glioblastoma
}

\author{
Raffaele Addeo $\cdot$ Michele Caraglia $\cdot$ M. Serena De Santi $\cdot$ Liliana Montella $\cdot$ \\ Alberto Abbruzzese - Ciro Parlato • Bruno Vincenzi - Marco Carraturo • \\ Vincenzo Faiola $\cdot$ Michele Genovese $\cdot$ Gregorio Cennamo $\cdot$ Salvatore Del Prete
}

Published online: 21 September 2010

(C) Springer Science+Business Media, LLC. 2010

\section{Erratum to: J Neurooncol}

DOI 10.1007/s11060-010-0329-z

The Abstract section of the original publication contains the following two errors:

- right column, lines 6-7: “...then every 3 weeks at $100 \mathrm{mg} / \mathrm{m}^{2}$..." should read "...then every 4 weeks at $80 \mathrm{mg} / \mathrm{m}^{2} \ldots "$.

The online version of the original article can be found under doi: 10.1007/s11060-010-0329-z.

R. Addeo $(\square) \cdot$ L. Montella $\cdot$ G. Cennamo · S. D. Prete Oncology Department, "S. Giovanni di Dio" Hospital, ASL Napoli2 Nord Via Giovanni XXIII, 80028 Frattaminore, Naples, Italy

e-mail: lelloaddeo@alice.it

M. Caraglia · A. Abbruzzese

Department of Biochemistry and Biophysics, Second University

of Naples, Via Costantinopoli, 16-80138 Naples, Italy

M. S. De Santi · M. Genovese

Neurosurgery Department, "Umberto I" Hospital ASL SA, V.

F. Ricco, 84014 Nocera Inferiore, Salerno, Italy

C. Parlato

Department of Neurosurgery, Second University of Naples,

Viale Colli Aminei 21, Naples, Italy

B. Vincenzi

Section of Oncology, Campus Biomedico University,

Rome, Italy

M. Carraturo

Radiotherapy Unit, ASL Napoli 2 Nord, Acerra, Naples, Italy

V. Faiola

Oncology Unit, AORN “'Ruggi D’Aragona” Hospital,

Salerno, Italy
- right column, lines 16-17: "Progression-free survival at 6 months was $61 \%$." should read "Progression-free survival at 6 months was 39\%.". 